\title{
A report of the Child and Adolescent Psychiatry Specialist Section
}

\section{Role of the child and adolescent psychiatrist}

The purpose of this paper is to describe the specific contribution of the child and adolescent psychiatrist to mental health services for those with responsibility for planning and administering such services.

The Royal College of Psychiatrists published a document on 'The Role, Responsibilities and Work of the Child and Adolescent Psychiatrist' in the Bulletin of the Royal College of Psychiatrists, 10, 202-206, August 1986 which sets out in detail the type of work done by the child and adolescent psychiatrist and the context in which this is most effectively implemented. That document sets out the broad range of activities expected of child and adolescent psychiatry and should be read in combination with the present paper in which the emphasis is on the training of child and adolescent psychiatrists and its relevance to their particular role in the health service. An understanding of the importance of child development is particularly important.

\section{Child mental health services}

A wide range of different disciplines are necessary for a comprehensive child mental health service. The disciplines most commonly involved are child and adolescent psychiatrists, clinical and educational psychologists, child psychiatric nurses, child psychotherapists, and psychiatric social workers. Each profession has unique experience, skills and knowledge specific to that profession and derived from the training they have received, but there are also certain areas of knowledge and skills in research, teaching and some treatment methods which are common to all or some of the professions.

\section{Training of the child and adolescent psychiatrist}

The specific role of the child and adolescent psychiatrist is based on their training and experience. Their training necessarily includes:

(1) General medical training and a basic medical qualification. Child and adolescent psychiatrists are registered medical practitioners.

(2) Post-qualification experience in various nonpsychiatric medical specialties: for example, paediatrics, obstetrics and gynaecology, general medicine and surgery.

(3) Specialist training in general psychiatry leading to the qualification of Membership of the Royal College of Psychiatrists (MRCPsych). Like other psychiatrists, child and adolescent psychiatrists are qualified to be registered as mental health officers and Section 12 approved doctors (Mental Health Act 1981).

(4) Higher Specialist Training in child and adolescent psychiatry. Training schemes are monitored and approved by the Joint Committee on Higher Psychiatric Training. Higher Training is four years so that the minimum period of training following basic medical qualification is not less than eight years.

This training experience provides a unique combination of knowledge and skill relevant to the assessment and treatment of psychiatric disorder in children and adolescents via:

(1) a grounding in scientific method and its application to research, assessment and treatment in mental and physical disorders

(2) a knowledge of the human body, its anatomy, physiology, biochemistry and pathology, including in particular an understanding of the wide range of links between mental and physical disorders. Training in normal and abnormal child development, psychology and sociology

(3) a basic knowledge of medicine in relation to the origins, diagnosis and treatment of physical disorder

(4) experience of physical illness and death in childhood and adult life

(5) clinical skills relevant to the diagnosis and treatment of physical disorders in adults and children, with knowledge of the range of specialist investigations and assessments which may be appropriate

(6) knowledge of the epidemiology, aetiology and course of the full range of mental disorders in adults, children and adolescents

(7) experience of both mild and severe mental illness in children, adolescents, and adults including those where there is a threat to life, such as child abuse, affective disorders and anorexia nervosa

(8) knowledge, experience and clinical skills relevant to the assessment and treatment of the full spectrum of mental disorder in adults, 
children and adolescents, including in-patient treatment

(9) experience and skills in consultation, decision making and administration in multidisciplinary work. An understanding of the administrative structure of the NHS and ability to practice a specialty in both hospitals and in the community

(10) skills and experience in teaching a wide range of professionals working with children

(11) direct experience of research and knowledge of how to assess the research of others

(12) a knowledge of the law pertaining to children and experience in presenting child psychiatric evidence to courts.

\section{The relevance of the training}

This breadth and depth of training is relevant:

(a) to planning child mental health services

(b) to administering and coordinating in-patient, day patient and out-patient services

(c) to teaching and consulting other disciplines and agencies on the full range of child and adolescent mental health particularly when a very broad range of knowledge is required including knowledge of physical disorders and the full range of potential interventions.

In direct clinical work this combination of knowledge, skills and experiences is essential when emotional, behavioural and developmental disorder in children and adolescents:

(a) is associated, or may be associated, with physical disorder in the child or those with whom they live

(b) is associated with mental disorders among those with whom they live, especially parents

(c) is such that administration of drugs needs to be considered

(d) is associated with the ingestion of drugs

(e) is of severe degree

(f) is associated with a threat to the life of the child or other person with whom they are associated whether due to natural causes or family dysfunction (g) might require admission to hospital including admission under the Mental Health Act

(h) when a detailed and broad ranging assessment of a case is required, as when the presentation of a problem is complex or unusual, or when a wide range of aetiological factors are present: physical, emotional and social, or when there is doubt about the diagnosis

(i) where detailed coordination and decision making with respect to the case is required.

\section{Psychotherapeutic treatments}

There are now a wide range of psychotherapeutic treatments used with groups, families or individuals and employing behavioural, cognitive, psychodynamic or systemic approaches. It is often thought that the different professions offer different types of psychotherapy. It is assumed by many that clinical psychologists will automatically provide behavioural treatment, social workers family therapy and child psychiatrists psychodynamically orientated psychotherapy. This is not the case. Some major advances in behaviour modification have been made by psychiatrists and some child psychologists have received a full psychoanalytic training. Child psychiatrists receive an eclectic training and will have skill in a range of different forms of psychotherapy, as well as in other treatment modalities.

\section{Conclusions}

The child and adolescent psychiatrist has a training that is unique by virtue of the particular combination of knowledge, skills and experience which it contains, and which is not shared by any other profession. Other professionals working in the field of child mental health such as clinical child psychologists, child psychotherapists and psychiatric social workers have different but overlapping combinations of skills. A satisfactory child and adolescent mental health service requires a wide range of assessment and treatment provision, and this is only possible when contributions are available from the full range of relevant professionals. 